\title{
Design and Simulation of Two Channel QMF Filter Bank using Equiripple Technique.
}

\author{
Meena Kohli*, Rajesh Mehra** \\ *[M.E student, ECE Deptt ,NITTTR,Chandigarh, \\ **[Associate Professor, ECE Deptt , NITTTR,Chandigarh]
}

\begin{abstract}
In this paper a design of a two channel FIR QMF bank for perfect reconstruction is presented. The QMF design has multicriterion constraints such as minimal of reconstruction error and minimal of iterations are among the most important problems in filter bank design. The main problem of filter bank design is to find adequate coefficients for the prototype filter $H_{0}$ such that the prototype filter has minimum error in stopband, passband and transition band. The proposed QMF filter bank has been developed using equiripple linear phase FIR filters with MATLAB. The developed equiripple linear phase filter bank performance has been compared with kaiser window based filter bank in terms of peak reconstruction error. The results show that equiripple based filter bank provides better PRE ranging from $16.45 \%$ to $17.31 \%$ as compared to kaiser window based filter bank.
\end{abstract}

Keywords: Equiripple FIR filters, Kaiser Window, Perfect Reconstruction, QMF Filter bank.

\section{Introduction}

In many applications, a discrete time signal $\mathrm{x}[\mathrm{n}]$ is first split into a number of subband signals by means of analysis filter bank, the subband signals are then processed and finally combined by a synthesis filter bank resulting in an output signal. If the subband signals are band limited to frequency ranges much smaller than that of the original input signal, they can be down-sampled before processing. Because of the lower sampling rate, the processing of the down-sampled signal can be carried out more efficiently. After processing these signals are up-sampled before being combined by the synthesis bank into a higher rate signal. The combined structure applied is called quadrature mirror filter.

The QMF is a filter most commonly used to implement a filter bank that split an input signal into two bands. Initially, it was used for aliasing cancellation in subband coding of the speech signals. During the last two decades QMF bank have found various applications in several different areas such as sub band coding of speech signal, image processing, ECG signal Compression processing, A/D convertors and design of wavelet base.The multirate structure in which the output sequence is a delayed and scaled replica of input sequence is called perfect reconstruction multirate system. There are three types of distortions in QMF banks: aliasing distortion, phase distortion and amplitude distortion. The aliasing distortion is eliminated with use of suitable design of the synthesis filters and phase distortion is removed with use of a linear phase FIR filter. Amplitude distortion can be minimized using computer aided techniques or equalized by cascading with a filter [1].

Many researchers used a new iterative approach.The convex minimax optimization is used in [2] but has disadvantage that longer filter length is required. The condition for achieving a low-magnitude reconstruction error, required for Nearly Perfect Reconstruction CMFBs can be approximately satisfied. The design is formulated as a convex minimax or least-square (LS) optimization problem. The optimal solution used the nonlinear optimization using the Parks-McClellan algorithm, Second Order Cone Programming (SOCP), or SemiDefinite Programming (SDP) [3] and the linear optimization process achieve minimum value of reconstruction error near to perfect reconstruction using kaiser window based Prototype low pass filter [4]. The modified algorithm has been developed to optimize the reconstruction error using linear objective function through iteration method. Another approach is to use Differential Evolution (DE) and Fitness- Adaptive Differential Evolution (FiADE). DE has emerged as a powerful and robust tool for solving the linear and nonlinear equations [6], but has a drawback that stop band edge attenuation is less.

QMF is used for many applications like efficient method for the design of two-channel, quadrature mirror filter (QMF) bank for subband image coding [7]. The objective function is minimized directly, using nonlinear unconstrained method. The another approach to design QMF is to use polyphase components but it results in dramatic computational efficiency and reduced number of iterations but effective for large filter taps [9]. 


\section{QMF Bank}

The multirate digital structure that employs two decimator in the signal analysis section and two interpolators in the signal synthesis section is shown in fig1.

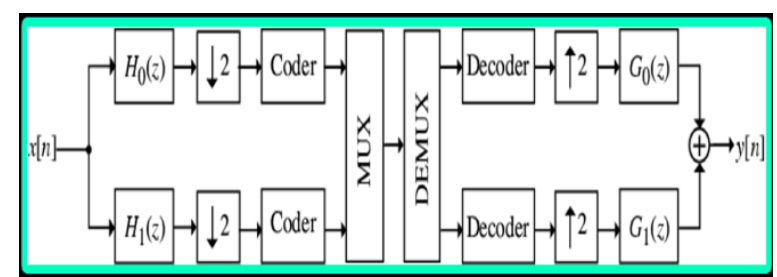

Fig. 1.General structure of a two channel QMF bank.

If the down-sampling and up-sampling factors are equal to or greater than the number of bands of the filter bank, then the output can be made to retain all of the characteristics of the input signal using proper filters structure. To obtain a Perfect Reconstruction (PR) or Near Perfect Reconstruction (NPR), the three distortions are eliminated or minimized using Alias-free filter bank, Linear phase FIR filter and Perfect reconstruction filter bank [6].

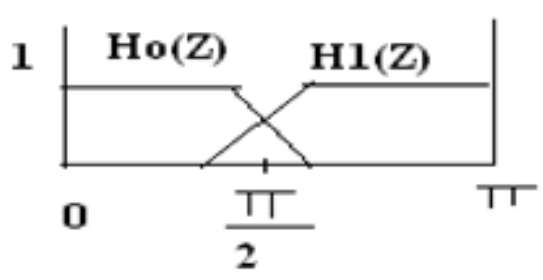

Fig. 2. Magnitude response of overlapping analysis filters.

The Fig.2 shows the filter response of the lowpass filter $\mathrm{H}_{0}(\mathrm{Z})$ and highpass filter $\mathrm{H}_{1}(\mathrm{Z})$. The $\mathrm{z}$ Transforms of the input signals are [8]

$\mathrm{X}_{0}(\mathrm{z})=\mathrm{X}(\mathrm{z}) \mathrm{H}_{0}(\mathrm{z})$

$\mathrm{X}_{1}(\mathrm{z})=\mathrm{X}(\mathrm{z}) \mathrm{H}_{1}(\mathrm{z})$

The output signals $Y_{0}[n]$ and $Y_{1}[n]$ are added to obtain the single output $y[n]$.

The $\mathrm{z}$-transform of $\mathrm{y}[\mathrm{n}]$ is given as

$\mathrm{Y}(\mathrm{z})=\mathrm{Y}_{0}(\mathrm{z})+\mathrm{Y}_{1}(\mathrm{z})$

$\begin{aligned} \mathrm{Y}(\mathrm{z})= & \frac{1}{2}\left[\mathrm{H}_{0}(\mathrm{z}) \mathrm{G}_{0}(\mathrm{z})+\mathrm{H}_{1}(\mathrm{z}) \mathrm{G}_{1}(\mathrm{z})\right] \mathrm{X}(\mathrm{z})+ \\ & \frac{1}{2}\left[\mathrm{H}_{0}(-\mathrm{z}) \mathrm{G}_{0}(\mathrm{z})+\mathrm{H}_{1}(-\mathrm{z}) \mathrm{G}_{1}(\mathrm{z})\right] \mathrm{X}(-\mathrm{z})\end{aligned}$

$\mathrm{Y}(\mathrm{z})=\mathrm{T}(\mathrm{z}) \mathrm{X}(\mathrm{z})+\mathrm{A}(\mathrm{z}) \mathrm{X}(-\mathrm{z})$

Where, $\mathrm{T}(\mathrm{Z})$ is the distortion transfer function and $\mathrm{A}(\mathrm{Z})$ is the aliasing distortion. The first term is a desired signal and the second term is because of effect of aliasing which is to be eliminated.

The alias-free design of the two-channel filter bank is achieved by elimination of the $\mathrm{A}(\mathrm{Z})$ aliasing distortion in equation (3).

To eliminate the effect of aliasing $\mathrm{A}(\mathrm{z})=0$

So, $\mathrm{H}_{0}(-\mathrm{z}) \mathrm{G}_{0}(\mathrm{z})+\mathrm{H}_{1}(-\mathrm{z}) \mathrm{G}_{1}(\mathrm{z})=0$

This condition is simply satisfied by selecting in the $\mathrm{z}$ transform,

$H_{0}(z)=H(z)$ 
$H_{1}(z)=H(-z)$

$G_{0}(z)=H(z)$

$G_{1}(z)=-H(z)$

These are conditions for perfect reconstruction.

With $\mathrm{A}(\mathrm{z})=0$, now consider the condition for which the output of QMF bank is identical to the input except for an arbitrary delay, for all possible inputs. When this condition is satisfied, the filter bank is called a Perfect Reconstruction QMF bank.

$T(z)=\frac{1}{2}\left[H_{0}(z) G_{0}(z)+H_{1}(z) G_{1}(z)\right]=Z^{-k}$

By making use of $(5)$

$H^{2}(z)-H^{2}(-z)=2 Z^{-k}$

The distortion transfer function of the two-channel analysis/synthesis filter bank satisfying the Perfect Reconstruction property is a pure delayed function.

$T(z)=Z^{-n 0}$

And the FIR two-channel filter bank with linear-phase analysis and synthesis filters will be perfect reconstruction type if $[8]$

$\left|H_{0}\left(e^{j w}\right)\right|^{2}+\left|H_{1}\left(e^{j w}\right)\right|^{2}=1$

The equation (7) cannot satisfied exactly due to finite length of filter so it always exhibit some amplitude distortion unless it is a constant for all value of $\omega$. The corresponding objective function is constructed using weighted sum of mean square of errors in these responses can be written as

$\Phi=\max \left\{\left|\mathrm{H}_{0}\left(\mathrm{e}^{\mathrm{jw}}\right)\right|^{2}+\left|\mathrm{H}_{1}\left(\mathrm{e}^{\mathrm{jw}}\right)\right|^{2}-1\right\}$

The objective function can be minimized using procedure given in [4].

The peak reconstruction error [11] is given as

PRE $=\max 20 \log _{10}\left\{\left|H_{0}\left(e^{j w}\right)\right|^{2}+\left|H_{1}\left(e^{j w}\right)\right|^{2}\right\}$

Thus the amplitude distortion mainly occurs in the transition band of these filters. The degree of overlap responses of low pass and highpass is very crucial in determining this distortion. This distortion can be minimized by controlling the overlap, which in turn can be controlled by appropriately choosing filter coefficients.

III. QMF Bank Design Simulation.

Kaiser window approach has been used to design prototype lowpass filter. Impulse response of lowpass filter $\mathrm{h}(\mathrm{n})$ of length $(\mathrm{N}+1)$ is given by equation $(12)[4,6]$

$h(n)=h_{i}(n) w(n)$

Where

$\mathrm{h}_{\mathrm{i}}(\mathrm{n})=\sin \left(\mathrm{w}_{\mathrm{c}}(\mathrm{n}-0.5 \mathrm{~N})\right) / \pi(\mathrm{n}-0.5 \mathrm{~N})$

Is the impulse response of the ideal filter with cutoff frequency $\omega_{c}$ and the Kaiser window function can be specified by the three parameter $\omega_{\mathrm{c}}, \mathrm{N}$ and $\beta$.

$\mathrm{w}(\mathrm{n})=\mathrm{I}_{0}\{\beta(1-\mathrm{n}) / \mathrm{M}\} / \mathrm{I}_{0}(\beta) \quad-\mathrm{M}<\mathrm{n}<\mathrm{M} \quad(14)$ 
Where $\beta$ is the window shape parameter which control the stopband attenuation and is given as

$$
\beta=\left\{\begin{array}{cc}
0 & \text { for As }<21 \\
0.5842(\text { As }-21) \wedge 0.4+ & 0.07886(\text { As }-21) \\
0.1102(\text { As }-8.7) & \text { for } 21 \leq \text { As } \leq 50 \\
\text { for As }>50
\end{array}\right.
$$

and $\mathrm{I}_{0}(\beta)$ is modified zero ${ }^{\text {th }}$ order Bessel function. The order of the window $\mathrm{N}$ can be estimated by the following equation.

$$
\mathrm{N}=2\left(\mathrm{~A}_{\mathrm{s}}-7 \cdot 95\right) /(4 \cdot 36 \times \Delta \mathrm{w})
$$

Where As is the stop band attenuation and $\Delta \omega$ is the normalized transition bandwidth.

For the design of kaiser window based prototype filter for $\omega \mathrm{s}=0.3 \pi$, As (Stopband attenuation) $=80 \mathrm{~dB}$, and length of filter $(\mathrm{N}+1)=61$ are considered and the filter coefficients are obtained and passband frequency $\omega_{\mathrm{p}}=0.13 \pi$ is calculated. The peak reconstruction error in $\mathrm{dB}$ is 0.0104 obtained using QMF design. The frequency response is shown in Fig. 4.

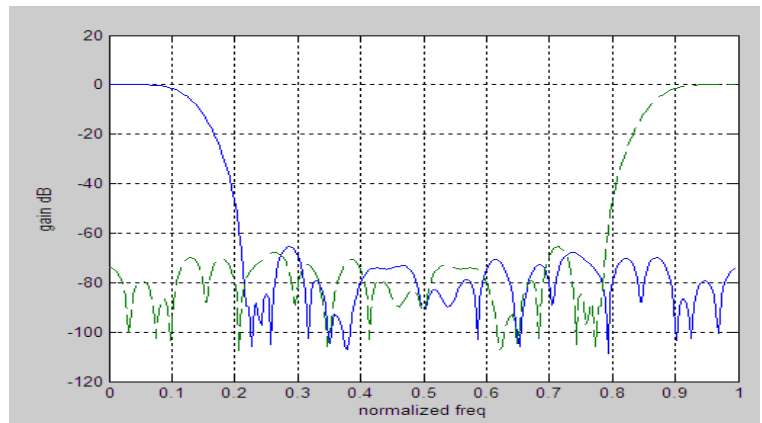

Fig.3 Frequency response of two channel FIR QMF Bank using Kaiser window of order 60.

The other specifications for the design of kaiser window based prototype filter are $\omega \mathrm{s}=0.3 \pi$, As (Stopband attenuation $)=90 \mathrm{~dB}$, and length of filter $(\mathrm{N}+1)=87$. The filter coefficients are obtained and $\omega_{\mathrm{p}}$ $=0.1605 \pi$ is calculated. The peak reconstruction error in $\mathrm{dB}$ is 0.0073 obtained using QMF design.

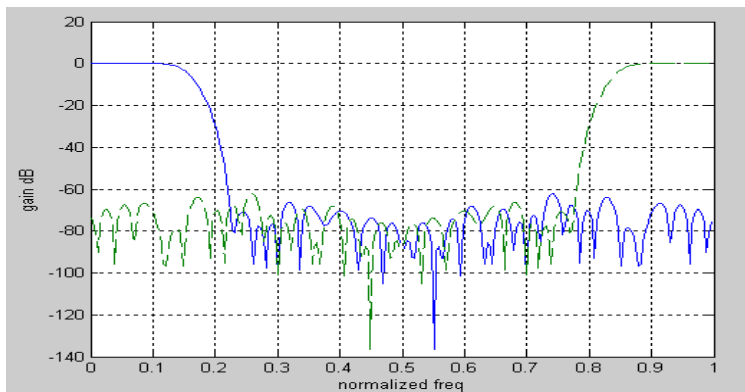

Fig.4 Frequency response of two channel FIR QMF Bank using Kaiser window of order 86.

Practical FIR designs typically consist of filters that meet certain design specifications, i.e., that have a transition width and maximum passband/stopband ripples that do not exceed allowable values. Linear-phase equiripple filters are desirable because they have the smallest maximum deviation from the ideal filter when compared to all other linear-phase FIR filters of the same order. For the design of Equiripple based prototype filter for $\omega \mathrm{s}=0.3 \pi$, As (Stopband attenuation) $=80 \mathrm{~dB}$, and length of filter $(\mathrm{N}+1)=61$ the filter coefficients are obtained and is $\omega_{\mathrm{p}}=0.13 \pi$ is calculated. The peak reconstruction error in $\mathrm{dB}$ is .0086 obtained using QMF design. 


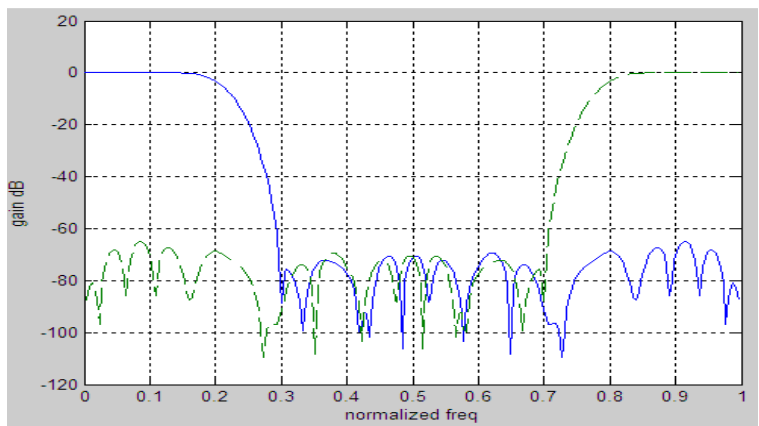

Fig.5 Frequency response of two channel FIR QMF Bank using equiripple filter of order 60.

The other specifications considered for the design of Equiripple based prototype filter are $\omega \mathrm{s}=0.3 \pi$, As $($ Stopband attenuation $)=90 \mathrm{~dB}$, and length of filter $(\mathrm{N}+1)=87$. The filter coefficients are obtained and $\omega_{\mathrm{p}}$ $=0.1605 \pi$ is calculated. The peak reconstruction error in $\mathrm{dB}$ is 0.0061 obtained using QMF design.

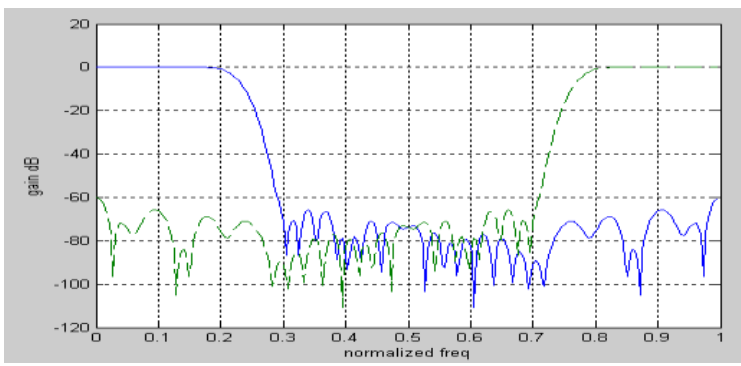

Fig.6 Frequency response of two channel FIR QMF Bank using equiripple filter of order 86.

\section{Results and Discussions}

The filters designed with the Kaiser window and with the remez function. Clearly the maximum deviation is smaller for the remez design and the performance is evaluated in terms of Peak Reconstruction Error (PRE) and stop band attenuation. In fact, since the filter is designed to minimize the maximum ripple, an equiripple filter can be designed that is superior to the Kaiser-window design. The simulations are carried out using MATLAB.

Table1. Comparison of result

\begin{tabular}{|l|l|l|l|}
\hline Method & As $(\mathrm{dB})$ & Filter length & $\begin{array}{l}\text { PRE in } \\
\%\end{array}$ \\
\hline $\begin{array}{l}\text { Kaiser window } \\
\text { existing method }\end{array}$ & 80 & 61 & 0.0104 \\
\hline $\begin{array}{l}\text { Equiripple filter } \\
\text { proposed method }\end{array}$ & 80 & 61 & 0.0086 \\
\hline $\begin{array}{l}\text { Kaiser window } \\
\text { existing method }\end{array}$ & 90 & 87 & 0.0073 \\
\hline $\begin{array}{l}\text { Equiripple filter } \\
\text { proposed method }\end{array}$ & 90 & 87 & 0.0061 \\
\hline
\end{tabular}

The table1 shows the comparison of two methods, Kaiser window and equiripple in terms of different parameter and comparison char is drawn for both methods.

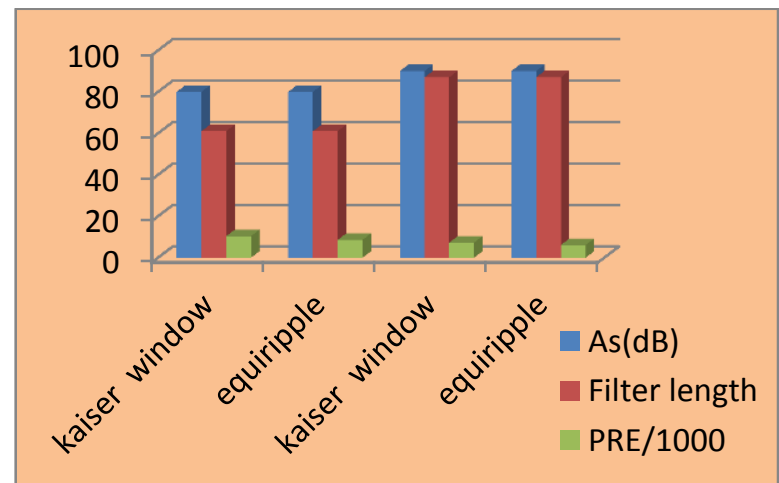

Fig.7 Comparison chart of table1. 


\section{Conclusion}

The equiripple filters are desirable because they have smallest deviation from ideal filters.The PRE is small in case of equiripple filters when compared with kaiser window based filters.It can be effectively used with filters of larger taps. The MATLAB based results show that equiripple based filter band provide better PRE 0.0086 for filter order 60 and 0.0061 filter order 86 compared to kaiser window based filter band 0.0104 filter order 60 and 0.0073 for filter order 86.

\section{References:}

[1] S. C. Chan, C. K. S. Pun, K. L. Ho,"New design and realization techniques for a class of perfect reconstruction two-channel FIR filter banks and wavelet bases", IEEE Transactions Signal Processing, Vol. 52, No. 7, Pp. 2135-2141, 2004.

[2] R. Bregovic and T. Saramaki, "A general-purpose optimization approach for designing two-channel FIR filter banks", IEEE Transactions on signal processing, vol. 51, no. 7, , Pp. 3303-3314, July 2003.

[3] S. C. Chan and S. S. Yin, "On the design of low delay nearly-PR and PR FIR cosine modulated filter banks having approximate cosine-roll off transition band", in Proc. EUSIPCO, Pp. 1253-1256 ,Sep. 2004.

[4] Ram Kumar Soni, Alok Jain, and Rajiv Saxena, "New Efficient Iterative Optimization Algorithm to Design the Two Channel QMF Bank", World Academy of Science, Engineering and Technology, Vol. 24, Pp 56-60, 2008.

[5] T Ghosh, A.; Giri, R.; Chowdhury, A.; Das, S.; Abraham, A. "Two-Channel Quadrature Mirror Bank Filter Design Using a FitnessAdaptive Differential Evolution Algorithm", Nature and Biologically Inspired Computing (NaBIC), Second World Congress , Pp $634-641,2010$.

[6] Sanjit.K.Mitra, "Digital Signal processing", Tata McGraw-Hill Edition 1998.

[7] Anamika Jain, Aditya Goel , "Unconstrained Optimization Method to Design Two Channel Quadrature Mirror Filter Banks for Image Coding", International Journal of Image Processing (IJIP), Vol. 5 Issue 1, Pp. 65-71, March 2011.

[8] Sanjit.K.Mitra, "Digital Signal processing: A Computer based Approach", Tata McGraw-Hill Edition 2001 Ch. $7 \& 10$.

[9] Ankita Gupta, S.K. Agarwal, "Designing of Two Channel Polyphase Quadrature Mirror Filter Bank using Power Optimization Method "Proceeding International Conference on Computer and Communication Technology, Pp 280-284, 2011.

[10] S. S. Yin, S. C. Chan, Member, IEEE, and K. M. Tsui,\|l On the Design of Nearly-PR and PR FIR Cosine modulated Filter Banks Having Approximate Cosine-Rolloff Transition Band, IEEE transactions on circuits and systems, Volume. 55, No. 6, Pp-571-575, June 2008 .

[11] Suverna Sengar and Dr. Partha Pratim Bhattacharya, "Design and Performance Evaluation of a Quadrature Mirror Filter (QMF) Bank”, Proceeding International Journal of Electronics \& Communication, Vol. 3, Issue 1, Pp- 209-212, March 2012. 